\title{
La peur de l'impensable dans l'histoire des sciences : le cas de la science juridique
}

Gérard Timsit

\section{OpenEdition}

Journals

Édition électronique

URL : http://journals.openedition.org/ress/673

DOI : $10.4000 /$ ress.673

ISSN : 1663-4446

Éditeur

Librairie Droz

Édition imprimée

Date de publication : 1 décembre 2000

Pagination : 79-88

ISBN : 2-600-00481-5

ISSN : 0048-8046

\section{Référence électronique}

Gérard Timsit, «La peur de l'impensable dans l'histoire des sciences : le cas de la science juridique », Revue européenne des sciences sociales [En ligne], XXXVIII-119 | 2000, mis en ligne le 15 décembre 2009, consulté le 04 mai 2019. URL : http://journals.openedition.org/ress/673 ; DOI : 10.4000/ ress.673 


\section{Gérard TIMSIT}

\section{LA PEUR DE L'IMPENSABLE DANS L'HISTOIRE DES SCIENCES : LE CAS DE LA SCIENCE JURIDIQUE}

Je crains qu'il ne soit guère possible, dans cette réflexion sur la peur de l'impensable, de donner à penser un système théorique comme déjà établi, une orthodoxie comme déjà formée - et la peur comme le sentiment d'un danger intellectuel que l'on éprouverait à transgresser les limites du système ou à échapper aux règles de l'orthodoxie - sans que l'on ait à s'interroger sur ce qui fait la légitimité du système ou les fondements de l'orthodoxie. Je craindrais que, ce faisant, l'on ignore l'une des dimensions du problème de la peur de l'impensable dans la science juridique - qui est une science sociale.

La peur de l'impensable en droit, c'est en effet, me semble-t-il - fondamentalement - la peur de ne plus trouver dans la pensée sur le droit les moyens de penser le droit comme moyen de se protéger contre la peur. Le droit - et c'est bien ainsi qu'il apparaît dans la formule que je viens d'énoncer - est à la fois, science et technique. Technique - technique sociale -, il est né de la peur. Il a été conçu dans des sociétés qui avaient peur, qui connaissent la peur, comme moyen d'y résister, de la vaincre ou de la supporter. L'exemple le plus clair, le plus caractéristique, que l'on en puisse donner est évidemment celui du droit pénal - celui des droits qui, de la manière la plus typique, est destiné à préserver la société contre les peurs, contre toutes les peurs, qui l'assaillent.

Les systèmes théoriques construits pour en rendre compte ont donc dû faire face à cette conception de la technique juridique et de la fonction sociale qui lui est reconnue. Cette conception fonctionnelle de la loi n'a pas pu ne pas constituer l'une des frontières de l'impensable. Sans doute une frontière peut-elle toujours être franchie, une limite toujours dépassée, mais cette pensée de la transgression, du «pas au delà » comme dit Maurice Blanchot, est toujours plus difficile: elle a donc dû être justifiée dans des conditions telles qu'elle ne pût porter atteinte à ce sentiment de l'irréparable que constitue, de la part de la science, la mise en cause de l'une des fonctions fondamentales, sinon de la fonction centrale, assignée à la technique dont la science prétend rendre compte. Les choses ne pourraient changer que le jour où l'on pourrait démontrer que cette fonction s'est transformée, ou qu'elle n'est pas la seule qui doive être reconnue. Or c'est bien ce qui paraît s'être produit, ou être en train de se produire, en droit - et semble permettre de rendre compte de la manière dont a changé la pensée sur le droit. Concevant le droit, lorsqu'il s'agissait de construire un système théorique, comme moyen de résister à la peur, ou au moins de la domestiquer, la pensée sur le droit n'a pu se transformer et connaître de vraie mutation que, lorsque cessant de voir dans le droit un moyen de contrainte - un moyen de faire peur destiné à résister à la peur -, elle a pu y découvrir ce qu'il est vraiment: non un instrument de sanction destiné à punir la 
violation de l'ordre établi ou la non-réalisation de la réalité désirée, mais plus généralement, une technique de traitement, et pour cela, de représentation de la réalité. On analysera donc, pour le montrer, d'abord la formation du système théorique dominant en droit (I), puis la mutation qu'il est en train de connaître (II).

\section{I. - LA FORMATION DU SYSTÈME THÉORIQUE DU DROIT}

Je veux ici prendre deux précautions avant de parler de la formation du système théorique du droit. La première, d'ordre historique, pour avertir que mon analyse ne portera que sur la formation du système théorique dominant du droit moderne - un système qui prend place à partir du milieu du $\mathrm{XIX}^{\mathrm{e}}$ siècle avec le positivisme. Il me semble cependant que mon analyse pourrait tout aussi bien trouver application dans la formation de systèmes chronologiquement antérieurs, le système jusnaturaliste, par exemple. La seconde précaution est d'ordre sémantique: si je parle d'un système théorique, c'est qu'il me semble en effet possible, malgré la diversité, et parfois l'opposition, des analyses ici recensées, de les réunir toutes dans le singulier d'un système, compte tenu de leur profonde unité fonctionnelle et de la manière dont, pour permettre au droit d'assumer la fonction qu'elles lui assignent, elles préservent, par différents amendements ou aménagements, quelques uns des traits les plus essentiels de l'architecture de la construction originelle.

Je voudrais donc d'abord analyser comment, pour répondre à la mission conférée au droit, la construction théorique en a été conçue dans ses débuts - sur quelles fondations elle repose -, puis par quelles transformations en a ensuite été assurée la perpétuation.

\section{La fondation du système théorique}

Tout le monde a été, est ou sera positiviste. Le positivisme en droit est une sorte de repère nécessaire de toute la théorie juridique - un passage obligé de toute réflexion sur le droit et le moyen sûr de discréditer toute analyse qui se verrait reprocher d'ignorer le droit positif. Les positivistes prétendent être seuls à connaître le droit - le jus positum, dont ils se sont arrogés le maniement - et l'intelligence. Je polémique à peine... Lorsqu'il y a quelques années, un recueil de textes consacrés au positivisme juridique ${ }^{1}$ est publié, on s'étonne - et finalement, on ne s'étonne plus guère ...- d'y voir qualifier de positivistes les analyses les plus diverses, défendues par des auteurs que, pourtant, l'on serait tenté de ranger dans les écoles ou mouvances doctrinales opposées. "A proprement parler, nous y explique-t-on, il n'y a pas de théorie du droit positiviste ». Il n'y a que des thèses «attribuées » au positivisme: les thèses les plus diverses. Il suffit que l'on adhère à l'une d'entre elles pour mériter le nom de positiviste. Quelles thèses? 1) Le droit est posé par une volonté humaine - le Législateur ou le juge. Et non pas Dieu ou la Raison. 2) Il est possible d'identifier les sources du droit: des sources formelles, dont l'origine est parfaitement identifiable: la législation, la jurisprudence - mais

Chr. Grzegorczyk, Fr. Michaut, M. Troper, Le positivisme juridique, Paris, LGDJ, 1992. 
pas la coutume ou les pratiques. 3) Le droit positif est le droit efficace - et, s'il est efficace, c'est qu'il a été produit par la volonté du Souverain, c'est-à-dire de celui qui est habituellement obéi. Mais qui est souverain? 4) Le droit est gouverné par la logique. Mais peu importe la logique qui le gouverne: formelle, syllogistique, déductive - ou non. 5) Le rôle du juge est d'interpréter et appliquer la loi. Mais on ne s'accorde guère sur la portée et les limites de l'interprétation et de l'application: le juge est-il, en effet, « la bouche de la loi », ne créant d'autre droit que celui que portent ou impliquent les dispositions de la loi qu'il est chargé d'appliquer, ou bien $\mathrm{a}-\mathrm{t}$-il un pouvoir normatif véritable au delà du texte, et parfois même contre lui? 6) Le positivisme est une doctrine étatiste. Mais l'étatisme du droit - le fait que l'Etat soit à l'origine du droit - est-ce une qualité du droit? Ou la source de son imperfection? Et enfin: 7) Le droit est un système; mais est-il un système unifié, un ensemble cohérent de règles dont l'interaction et l'unité sont assurées par leur soumission à un ou des principes communs? Ou bien un agrégat de normes hétérogènes? ...

Le positivisme, en définitive, n'est pas une théorie, mais un monde: une théorie-monde - un système de théories, un système des théories les plus diverses dont, en vérité, la seule unité, alors que ces théories relèvent des approches les plus opposées du droit - le droit, produit de l'industrie du Législateur (qui a élaboré la loi dont il est formellement l'auteur), ou bien résultat de la seule interprétation qu'en donne le Juge (chargé de la lire et, en définitive, de lui donner application ...) - réside dans la fonction qu'elles lui attribuent: une fonction de protection (ou de promotion) de l'ordre social: si tu violes l'ordre établi (ou si tu ne contribues pas à la mise en œuvre de l'ordre que j'établis), tu seras puni. La reconnaissance d'une telle fonction a deux implications, toutes deux liées à la peur - qui est la peur éprouvée du manquement à l'unité ou à la sécurité du groupe social.

A. La première implication a trait à la définition même du droit. Dans un tel système théorique, la loi, considérée comme produit de l'industrie du législateur ${ }^{2}$, est nécessairement définie comme obligation assortie d'une sanction ${ }^{3}$ - une sanction infligée par l'Etat en cas de violation de la norme imposant aux individus les obligations auxquelles ils sont soumis de la part du Souverain: respecter l'ordre instauré (ou s'intégrer à l'ordre promu) par l'Autorité. La sanction est, dans cette définition, partie intégrante du concept même de loi. On eût pourtant pu penser, et l'on n'a pas manqué de le faire, que la notion de loi n'exigeait ni que l'on définît la norme par référence à la sanction qui vient en punir la violation ni que l'on fît de la sanction le seul critère de la distinction entre normes juridiques et non-juridiques (morales, sociales ou religieuses ...). Deux raisons pouvaient plaider en ce sens : 1) on ne définit pas un objet par référence à un élément extérieur à l'objet: la loi est parfaite et complète dès qu'elle dispose; par là même, elle oblige; la sanction ne vient que de surcroît: elle est l'un des moyens - un seul parmi beaucoup d'autres, qui vont de l'obéissance spontanée à l'exécution forcée - de la mise

Cf. sur ce point l'analyse de M. Bastit, Naissance de la loi moderne, Paris, PUF, 1990.

Sur cette définition de la norme, et le présupposé qui la fonde, cf. G. Timsit, Les noms de la loi, Paris, PUF, 1991. 
en œuvre de l'obligation; 2) la définition de la loi par la sanction revient en fait à définir la loi par la loi elle-même puisque, quand il s'agit en effet de savoir quel type de sanction distingue la norme juridique des autres normes (sanctionnées, elles aussi : ainsi la violation de la norme sociale de politesse, par exemple, est-elle punie par la réaction du corps social qui vous tient désormais à l'écart du groupe...), il faut bien en passer, en dernière instance, par l'énoncé législatif de la sanction: on ne reconnaît une sanction juridique qu'à ce qu'elle est prévue par une norme juridique; et c'est justement la norme juridique qu'il s'agit de définir ... Cercle vicieux ...: incapacité théorique à définir la loi par la sanction qui vient en punir la violation.

Une telle incapacité témoigne d'autant mieux et d'autant plus des peurs dont la loi est censée protéger ceux qu'elle régit et abrite que cet illogisme théorique persistant à vouloir définir la loi par la sanction est doublé de ce constat, désormais assez général, que les lois ne consistent, en tout état de cause, pas seulement en commandements assortis de sanctions: la normativité ne se limite pas à la seule normativité impérative, la normativité «dure», contraignante; il en existe une autre, qui tient de plus en plus de place dans nos sociétés modernes. Elle s'analyse en actes de recommandations, de concertation, d'incitation ... - une normativité que l'on n'ose pourtant pas appeler, dans le positivisme ambiant, largement dominant, normativité ... On parle alors, pour la désigner, de normativité douce, de soft-law: normativité embryonnaire, quasi-normativité ... Une infra-normativité, vous dit-on. Et plus volontiers encore: une non-normativité ... ${ }^{4}$

Si c'était vrai et que rien ne méritât en effet le nom de loi qui ne fût assorti de sanction et donnât lieu à contrainte et violence, il faudrait donc admettre qu'il est de l'essence de la loi qu'elle fasse peur - pour protéger de la peur. Mais ce n'est pas vrai.

B. Cette conception de la loi comme instrument de protection de l'ordre social a en tout cas une autre implication dans la construction du système théorique destiné à en rendre compte. La norme est en effet conçue comme parfaite et complète dès qu'elle est inscrite dans le texte élaboré par le législateur. L'écriture de la loi est considérée comme la garantie indispensable, la protection la plus rigoureuse, que puisse offrir la loi à ceux qui en sont les destinataires et bénéficiaires. Lex, de: legere - c'est-à-dire: lire. On ne peut lire que ce qui est écrit. La loi est donc écriture. La loi moderne est née avec l'écriture. Et doit, en conclut-on, se tenir dans les limites de l'écriture. Le juge est la bouche qui prononce les paroles de la loi. Le jugement ne doit jamais être qu'un texte précis de la loi. On reconnaît là les formules célèbres de L'esprit des lois. L'écriture de la loi, que soit celle du législateur ou celle de la transcription qu'en fait le juge dans son jugement, apparaît ainsi comme condition de la liberté des individus - et la protection contre le mal et la peur de l'insécurité. L'Ecole de l'Exégèse - une sorte de quintessence du positivisme - porte ainsi jusqu'à la caricature cette conception de la loi: ne rien introduire dans le droit qui ne soit effectivement inscrit dans la loi ...; s'en tenir à la lettre de la loi.

$4 \quad$ Sur ce non-concept, cf. G. Timsit, Archipel de la norme, Paris, PUF, 1997. 
La scène de l'écriture chez les Nambikwara - la scène est racontée par LéviStrauss dans Tristes tropiques ${ }^{5}$ et commentée par Jacques Derrida dans sa Grammatologie $^{6}$, témoigne cependant de l'ambivalence de l'écriture 7 . L'écriture, condition de la liberté ? La loi - la loi écrite -, garantie et protection contre la peur et l'insécurité? On peut en douter. Et s'interroger, malgré l'intime association par le positivisme de la lettre et de la loi, sur l'effectivité d'une telle protection. La loi, parfaite et complète, parce que et dans la mesure où elle est écrite? Outre le fait que l'écriture de la loi fait place et donne lieu à l'indétermination de la norme une indétermination, condition de la liberté, certes, mais aussi occasion et moyen de la tyrannie, du fait de la liberté (de la discrétionnarité ?...) qu'elle offre au lecteur de la loi d'en dire le contenu -, outre ce fait, on ne peut en effet que constater la manière dont dérivent et dérapent les pratiques de l'écriture de la loi. Ce que l'on a appelé l' « inflation normative », la « logorrhée législative et réglementaire» en témoignent de manière inquiétante: «la loi jetable n'est pas respectable», a-ton affirmé ${ }^{8}$. Toutes les lois, désormais, sont des lois jetables. Des lois écrites - et mille fois réécrites. Des lois sur le papier. Des tigres de papier ...

\section{La perpétuation du système théorique}

La perpétuation d'un système qui fût, autant que l'était le système théorique construit et fondé par les positivistes, à l'écart des réalités empiriques du droit ou en contradiction avec elles ne pouvait donc être assurée qu'au prix d'aménagements et amendements destinés, tout en rendant le système théorique plus conforme à la réalité qu'il s'agissait de restituer, à préserver la fonction de protection de l'ordre social que l'on avait conçue pour lui.

A. Les amendements, ce sont ceux qu'apporte le nouveau positivisme - celui d'un Herbert Hart ${ }^{9}$, par exemple - à la conception traditionnelle de la règle comme obligation assortie d'une sanction. «L'idée de règle, admet Hart, n'est nullement une idée simple». L'ordre juridique, ajoute-t-il, est une «union complexe de règles primaires et secondaires ». Les règles primaires, ce sont, dans l'analyse hartienne, les règles classiques - celles qui s'imposent et voient sanctionner leur violation. Les règles secondaires, quant à elles, sont des règles auxiliaires, "parasitaires » par rapport aux premières. Leur rôle est de permettre aux premières de fonctionner et s'appliquer mieux que si elles n'existaient pas: règles de reconnaissance, de changement, d'adjudication qui servent à déterminer avec exactitude, à adapter quand c'est nécessaire, à mettre en œuvre avec efficacité les règles primaires. Avec une telle analyse, Hart amende habilement la doctrine positiviste traditionnelle. Il admet l'existence de règles autres que celles qui, destinées à faire peur, sont destinées à protéger de la peur. Mais il ne cède rien sur l'essentiel de la

\footnotetext{
5 Cl. Levi-Strauss, Tristes tropiques, Paris, Plon, 1955.

6 J. Derrida, De la grammatologie, Paris, Ed. de Minuit, 1967.

7 Sur l'ambivalence de l'écriture juridique, condition ou non de la liberté, cf. G.Timsit, Les figures du jugement, Paris, PUF, 1993, pp. 74 ss.

$8 \quad$ Conseil d'Etat, Rapport public, Paris, La documentation française, 1991.

9 H. Hart, Le concept de droit, Bruxelles, Publications des Facultés universitaires Saint-Louis, 1992.
} 
fonction de protection du droit contre la peur: les règles destinées à protéger le corps social restent en effet au centre de son système théorique, et même en deviennent le noyau dur; les règles autres ne sont jamais que «secondaires », auxiliaires - Hart ne dit-il pas « parasitaires »? - des premières ...

B. Les aménagements du système théorique vont plus loin. Les thèses de Ronald Dworkin ${ }^{10}$, au delà du positivisme, en sont un bon exemple. Elles consistent, elles aussi, à conserver au droit sa fonction de protection de l'ordre social. Mais - admettant, conformément en cela aux thèses de l'école réaliste, que la charge en repose non plus sur le Souverain auteur de la loi, en mesure, parce qu'il est le Souverain, d'user de la force et des moyens de faire peur, mais sur le Juge, lecteur de la loi -, elles imposent au juge de lire la loi qu'il doit mettre en oeuvre à la lumière des valeurs de justice et d'équité - Dworkin parle de «droit-intégrité » - qui fondent le fonctionnement et assurent la cohérence du groupe social. Le droit reste investi, dans l'analyse dworkinienne, de sa fonction de protection contre la peur - la peur des autres, la peur du changement, la peur de l'insécurité, la peur de tout. Mais ce sont, dans le système théorique qui doit en rendre compte, d'autres éléments que ceux directement liés aux moyens de contrainte destinés à faire peur qui en assurent désormais la fonction. Ce n'est plus la sanction qui est au centre de la définition dworkinienne du droit, mais les valeurs qui fondent le droit - et font ainsi l'unité du corps social. L'analyse dworkinienne reste dans ces conditions, en dépit de ses aménagements au système théorique originel et de son éloignement du système théorique positiviste, à l'intérieur des limites du système dominant - fondamentalement ancrée dans l'idée que la fonction du droit est d'abord, sinon uniquement, une fonction de protection de l'ordre social - une fonction de protection contre la peur de sa désintégration ...

\section{II. - LA MUTATION DU SYSTÈME THÉORIQUE DU DROIT}

La mutation du système théorique du droit n'intervient qu'à partir du moment où l'on comprend que la fonction du droit n'est pas d'abord ni seulement de protéger l'ordre social - une fonction de protection contre la peur - mais plus largement, et en exécution de la volonté politique, une fonction de traitement de la réalité.

C'est en effet cette fonction de traitement de la réalité qui peut permettre de rendre compte de la généralité des missions dévolues au droit. Parler, comme on l'a fait jusqu'à présent, de fonction de protection de l'ordre social, et ne vouloir, dans le droit, voir que cette fonction ne peut que limiter abusivement le champ de la normativité - la réduire à la seule part de la normativité classique, la normativité impérative, bien en deçà de toutes les formes d'activité qu'intuitivement, aujourd'hui, l'on perçoit comme normatives et juridiques. Fonction de traitement de la réalité, qu'est-ce à dire ? - ceci que la loi est l'outil, le relais nécessaire, dont dispose l'autorité publique pour agir sur la réalité qu'elle veut, de quelque

10 R. Dworkin, PUF, L'empire du droit, Paris, PUF, 1994 
manière, conserver, modifier, protéger, orienter, sauvegarder, bouleverser ... Ainsi l'autorité publique qui veut agir sur la réalité doit-elle, avant de pouvoir agir, représenter - se donner une représentation - de la réalité sur laquelle agir: se donner une sorte de schéma descriptif qui, permettant de caractériser la situation à traiter, indique ceux des éléments qui devront en être transformés, conservés, supprimés, etc. Une telle représentation de la réalité ne peut jamais être que partielle: on ne saurait reproduire fidèlement, raconter exactement la réalité - ou bien la reproduction se confondrait avec la réalité elle-même. Tâche impossible. La loi, outil de traitement de la réalité, ne peut donc jamais être qu'un double infidèle de la réalité: juste une image approximative pour en assurer le traitement: une sorte de fantôme - comme on parle de fantômes, dans les bibliothèques, pour désigner ces plaquettes de bois ou de carton disposées sur les rayonnages, à la place des livres empruntés, pour en faciliter le repérage et le récolement ...

Se référer à une fonction de traitement de la réalité par la loi n'implique donc aucun a priori sur la mission du droit dans son rapport à la peur. Et c'est à partir du moment où est admise une telle mission - et que cesse la peur - que peut commencer la véritable mutation du système théorique. Ce qui était en effet au cœur de la définition de la loi dans le système théorique antérieur, c'était son caractère contraignant - et la sanction destinée à en punir la violation: une obligation assortie d'une sanction, disait-on... Désormais, on met l'accent, dans le nouveau système théorique, sur la relation de signification en laquelle elle s'analyse essentiellement. Changement de cap. Apparition de l'herméneutique. Le droit est d'abord affaire d'interprétation - avant même d'être une affaire de contrainte. Il en résulte une double révolution.

1. Dans les formes de l'activité normative, d'abord. A partir du moment où l'on admet du droit qu'il est affaire d'interprétation et relation de signification, on change de système, et au lieu que le droit doive être considéré comme fonctionnant sur le seul modèle classique d'un droit/réglementation abstrait et impersonel, il est désormais admis que le droit fonctionne sur un tout autre registre: celui de la régulation. Deux corps du droit, là où l'on n'avait jamais su en voir qu'un seul qui ont des caractéristiques fondamentalement opposées. Deux corps qui, dans la lignée de la métaphore des Corps du Roi analysée par Kantorowicz ${ }^{11}$, relèvent, l'un, de l'abstraction la plus pure - Kantorowicz parle de corps mystique -, l'autre du réel et du concret le plus absolu - il parle de corps naturel... On connaît, sur ce point, le thème de Kantorowicz. L'Etat moderne, dit-il, est né de la distinction qu'a su faire la pensée médiévale entre les deux corps du roi ${ }^{12}$. C'est à partir du

\footnotetext{
E. Kantorowicz, Les Deux Corps du Roi, Paris, Gallimard, 1988.
}

12 «... Car le Roi a en lui deux Corps, c'est-à-dire un Corps naturel et un Corps politique. Son Corps naturel, considéré en lui-même, est un corps mortel, sujet à toutes les infirmités qui surviennent par Nature ou par Accident, à la faiblesse de l'enfance ou de la vieillesse, et aux déficiences semblables à celles qui arrivent aux corps naturels des autres gens. Mais son Corps politique est un Corps qui ne peut être vu ni touché, consistant en une société politique et un gouvernement, et constitué pour la direction du peuple et la gestion du Bien public, et ce Corps est entièrement dépourvu d'Enfance, de Vieillesse et de tous autres défauts et faiblesses naturels auxquels est exposé le Corps naturel, et pour cette raison, ce que fait le Roi en son Corps politique ne peut être invalidé ou annulé par une quelconque incapacité de son corps naturel » (Edmund Plowden, Commentaries or Reports, cité par Ernst Kantorowicz, loc. cit., pp. 21-22). 
moment où la doctrine, dans les monarchies de l'Occident chrétien, entre $\mathrm{X}^{\mathrm{e}}$ et $\mathrm{XVII}^{\mathrm{e}}$ siècle, a pu opérer la dissociation du corps naturel du roi - affecté par la maladie ou anéanti par la mort - et de son corps politique ou mystique qui survivait aux maux et à la mort qui pouvaient frapper la personne du Prince que s'est installée l'idée d'Etat. Les formules, «Le roi ne meurt jamais », «Le roi est mort! Vive le roi !», rendent compte, dans leur brutale simplicité, de cette étrange alchimie théologico-politique présidant à la survivance du corps mystique au corps naturel - et, par delà la mort du roi, de cette persévérance de l'Etat dans son être moral et abstrait - bien que l'être concret et physique qui l'incarnait eût disparu. Ainsi est né l'Etat - de cette transmutation, extraordinairement racontée et disséquée par Kantorowicz, du corps naturel en corps mystique du roi - de son corps naturel accompli en corps politique.

Mais tandis que Kantorowicz perçoit cette naissance comme un processus de transformation du corps naturel en corps mystique - une transmutation de son corps physique et concret en un corps abstrait et désincarné, le processus de modernisation du droit semble devoir désormais être reconnu par la doctrine comme le résultat d'une évolution symétrique et inverse: de passage d'un droit abstrait, général et désincarné, expression d'un Etat lui-même héritier du corps mystique du roi, à un droit concret et ancré dans la réalité la plus physique et la plus sensible de la Société. Lorsque l'Etat moderne est apparu, il a en effet trouvé sa traduction dans un droit qui conservait des origines historiques de son Auteur, l'Etat, le caractère unitaire, mystique et abstrait dont celui-ci était paré. C'est ce droit-là qui a été le premier corps du droit - un droit impersonnel, général et désincarné. Je l'ai appelé: droit-réglementation. C'est sur ce droit-là, avec ce droit-là, qu'ont vécu longtemps, et encore maintenant, le plus largement, les Etats modernes. Mais ce droit a fait son temps. Abstrait et désincarné, il ne répond plus aux exigences de la gestion des sociétés post-modernes. Trop complexes pour être gérées aussi généralement, aussi impersonnellement et, pour ainsi dire, d'aussi loin, elles requièrent un autre droit - actuellement en développement - qui se caractérise, au contraire, par son adaptation au concret, son rapprochement des individus, son adéquation au contexte exact des sociétés qu'il prétend régir. Il est affaire d'interprétation - d'interprétation constante et constamment renouvelée. Concret, individualisé, contextualisé, c'est un droit de régulation.

Le problème est que de ces deux corps du droit - de réglementation et de régulation -, le second n'a été, jusqu'à présent, ni connu ni vraiment reconnu. Analysée, réduite et fondue, à peine apparue, dans les catégories traditionnelles de l'activité normative - droit ou non-droit -, la régulation ne s'est vu pendant longtemps conférer aucun statut autonome: ni en droit positif - aucune valeur juridique...ni a fortiori, en tant que concept de la science juridique. Ou bien, - pensait-on, en présence de l'une ou l'autre des formes d'activité normative des autorités publiques -, l'on avait affaire à du droit. Et ce ne pouvait être que de la réglementation. Ou bien, s'il ne s'agissait pas de réglementation, il ne s'agissait pas non plus de droit: ce ne pouvait être, tout au plus, qu'une activité de commentaire ou d'interprétation du droit. Du non-droit. La régulation était un non-concept.

Aujourd'hui, elle acquiert cependant un droit à l'existence ${ }^{13}-$ et emporte une deuxième révolution...

13 ... dont j'ai essayé de montrer l'émergence conceptuelle dans le droit français (cf., en particulier, le chapitre intitulé: «Naissance d'une notion», dans mon ouvrage Archipel de la norme, Paris, 
2. Dans la conception même du processus normatif. C'était un processus qui, originellement, et conformément aux idées héritées de la Grèce antique, était fondé sur une vision homogène de l'espace à la fois physique et politique. Coïncidence de deux modèles: un modèle cosmologique qui règle l'ordonnance de l'univers physique, et un modèle politique qui préside à l'organisation de la Cité. Dans les deux cas, un schéma spatial analogue, une même figure géométrique «où le centre seul a valeur privilégiée » et où, par conséquent, règnent à la fois les principes de hiérarchie et d'uniformité. De tels principes régissent des relations de caractère symétrique, réversibles et égalitaires. Idéal de l'isonomia - de l'égalité devant la loi - où tous, éléments du Cosmos et membres de la Cité, sont placés à égale distance du centre - le Soleil ou le Pouvoir -, et lui sont, de la même manière, soumis. L'abstraction et l'homogénéité de l'espace ainsi conçu trouvent leur traduction, au plan juridique, dans l'écriture de la loi, moyen de lier simultanément et uniformément l'ensemble des citoyens membres de la collectivité. «Mise sous le regard de tous par le fait même de sa rédaction, la formule écrite sort du domaine privé pour se situer sur un autre plan: elle devient bien commun, chose publique». L'écriture donne naissance à la Loi - la Règle, la Réglementation, forme synchronique et indifférenciée d'exercice du pouvoir.

Puis s'impose une nouvelle image du monde. Le monde des géomètres et des astronomes se sépare de celui de la Cité. «A la notion simple de l'égalité qui apparaissait dans l'idéal d'isonomia se substituent des conceptions plus savantes. On distingue, on oppose égalité arithmétique et égalité géométrique et harmonique. En fait, la notion fondamentale est devenue celle de proportion». De la Cité, «espace géométrique entièrement indifférencié», totalement homogénéisé sous l'autorité d'un Centre unique, on passe à la considération de la Société, composée d'individus divers et faite de situations différenciées. «L'espace de la Cité [...] s'oriente dans la voie d'une différenciation très poussée». Nouvelle image du monde qu'enregistre et reproduit aujourd'hui la nouvelle pensée de la loi. On y abandonne le modèle de la subsomption - une norme ne serait jamais que l'application stricte, par voie déductive et syllogistique, d'une norme supérieure édictée par le Souverain, autorité suprême: la loi n'était ainsi, dans ce modèle, que l'application de la constitution; la réglementation seulement la mise en œuvre rigoureuse de la loi, et le juge lui-même la bouche qui prononce les paroles de la loi pour un modèle nouveau: de déclinaison. La pensée de la régulation suppose en effet cette révolution - que de la Cité l'on soit passé à la Société; de l'égalité et de l'uniformité à une autre forme de l'égalité, plus universelle, parce que moins abstraite et plus différenciée, c'est-à-dire mieux adaptée à chacun des individus - et que l'on renonce à la conception de la Loi comme écriture générale et impersonnelle. Sans doute la Loi est-elle écriture. Mais elle exige pour son application Ricoeur dirait: pour son «effectuation ${ }^{14}-$ d'être relayée par une parole, celle de

PUF, 1997) et dont témoigne, par exemple, le plus récemment, l'utilisation, avec - semble-t-il toutes les caractéristiques de ce nouveau droit/régulation, du concept de co-régulation dans le domaine d'Internet.

14 Sur cette notion, cf. P. Ricoeur, Du texte à l'action, Paris, Seuil, 1986, p. 153. On se souvient de ses analyses qui assignent le même statut à la lecture et à la parole: «...on veut souligner le caractère «actuel» de l'interprétation: la lecture est comme l'exécution d'une partition musicale; elle marque l'effectuation, la venue à l'acte, des possibilités sémantiques du texte [...]. Ce caractère 
l'administrateur ou du juge, qui vient en singulariser, en contextualiser l'exécution en chacune des situations auxquelles elle doit être appliquée. Le pouvoir normatif, alors, ne se traduit plus dans une réglementation uniforme imposée du Centre et à tous également - contrainte, impérativité, extériorité de la loi traditionnelle qui s'accomplit dans l'instant même de son édiction. Ce pouvoir s'exerce dans la durée, formant un procès destiné à ne trouver d'achèvement que dans l'application concrète de normes générales aux situations singulières qu'elles viennent régir. Forme différée de l'exercice du pouvoir normatif, particulièrement adaptée à la fonction qui, désormais, lui incombe en priorité dans les sociétés modernes - une fonction de traitement des situations d'incertitude. En rupture avec la logique statocentrique - logique de l'Etat; logique statique -, de l'application uniforme d'une loi générale et impersonnelle, elle devient pour l'essentiel, et à cause même de l'environnement dans lequel et sur lequel elle agit, pouvoir de déclinaison - au sens le plus grammatical du terme: pouvoir de déclinaison au cas par cas des orientations générales inscrites dans une norme, à l'occasion de chacune des situations singulières, concrètes, auxquelles est confronté le titulaire du pouvoir normatif. Ce pouvoir de régulation ne peut donc être limité ni à l'édiction de la norme première comportant l'énoncé des orientations générales, ni aux normes secondes édictées dans le cadre de la norme générale. Ces «normes», considérées isolément, ne peuvent être assimilées ni traitées en normes classiques. Elles constituent, ensemble, l'exercice d'un seul et même pouvoir normatif - qui n'a d'existence que totale. Ou nulle. Il n'est complet, ce pouvoir, que dans ses deux temps, associés, et ses deux dimensions, indissociables. Révolution théorique qui place le droit à l'opposé des conceptions que l'on s'en faisait traditionnellement.

Ainsi se forme la nouvelle pensée du droit. Une pensée qui, brisant avec toutes les peurs, ouvre les voies d'une nouvelle réflexion sur les moyens d'endiguer la peur - et qui, au lieu de faire du droit un outil de contrainte pesant sur tous, en fait un instrument de la confiance de tous.

\section{Université de Paris I}

d'effectuation, propre à l'interprétation, révèle un aspect décisif de la lecture, à savoir qu'elle achève le discours du texte dans une dimension semblable à celle de la parole. Ce qui est ici retenu de la notion de parole, ce n'est pas qu'elle soit proférée; c'est qu'elle soit un événement, un événement du discours, l'instance de discours, comme dit Benveniste. Les phrases du texte signifient hic et nunc. Alors, le texte «actualisé » trouve une ambiance et une audience: il reprend son mouvement, intercepté et suspendu, de référence vers un monde et des sujets. Ce monde, c'est celui du lecteur; ce sujet, c'est le lecteur lui-même. Dans l'interprétation, dirons-nous, la lecture devient comme une parole. Je ne dis pas: devient parole. Car la lecture n'équivaut jamais à un échange de paroles, à un dialogue; mais la lecture s'achève concrètement dans un acte qui est au texte ce que la parole est à la langue, à savoir événement et instance de discours ». 